\title{
Infection control team activity and recent antibiograms in the Kobe University Hospital
}

\author{
Katsumi Shigemura ${ }^{1,2}$, Kayo Osawa ${ }^{2}$, Akira Mukai², Goh Ohji², Jong Ja Lee ${ }^{2}$, Hiroyuki Yoshida ${ }^{2}$, \\ Masato Fujisawa ${ }^{1}$ and Soichi Arakawa ${ }^{1,2}$
}

The hospital infection control team (ICT) has a major role in suppressing or preventing infectious diseases. The purpose of this study was to investigate whether the work of the ICT had affected the antibiograms at the Kobe University Hospital in the past 3 years. The ICT's works are as follows: (1) to monitor whether physicians are instructed in the use of broad-spectrum antibiotics; (2) to check whether measures for preventing the occurrence or spread of infectious disease are performed along with appropriate standard precautions; (3) to provide rapid communication with physicians in bacteremia cases and (4) reporting the antibiograms in the hospital. In addition, we investigated changes in the antibiograms every 6 months based on all materials. There were 193 physician interventions in 2010 and 491 in 2011 . The representative isolated bacteria included no additional bacteria with lower susceptibilities found over the past 1.5 years compared with the initial 1.5 years in the 3-year investigation period. The ratio for performing two sets of blood culture tests in all blood culture tests showed an upward tendency from $58.1 \%$ in 2009 to $71.1 \%$ in 2010 and $80.3 \%$ in $2011(r=0.995, P=0.063, b=0.089)$. In conclusion, since the introduction of an expanded ICT role, our data showed an increased antibiotic susceptibilities in bacteria such as Enteroccus faecalis and the total amount of hand disinfectant agents tended to increase year by year, even though direct statistical analyses could not easily be performed. Further observation may be necessary for a definitive evaluation of ICT activities.

The Journal of Antibiotics (2013) 66, 511-516; doi:10.1038/ja.2013.36; published online 1 May 2013

Keywords: antibiogram; infection control team; isolation of resistant strains

\section{INTRODUCTION}

New ways of thinking about infection and infectious disease have recently spread. Traditionally in the field of infection and/or infectious disease, treatments were considered to be well established and research focused on the exploitation of antibiotics. ${ }^{1}$ More recently, research has demonstrated that inappropriate use of antibiotics is the predominant factor in the spread of antimicrobial drug resistance. .,3 $^{2,}$

The control of hospital-acquired infections is a major priority for health-care organizations in developed countries, and evidence shows that an institutional approach is needed to reduce the incidence of infections. ${ }^{4}$ An important part of the work of the infection control team (ICT) is the formulary restriction and pre-authorization of specified broad-spectrum antibiotics such as carbapenems. ${ }^{5}$

Many universities and general hospitals have now established ICTs, and their activity range has been wide. ${ }^{5,6}$ In Japan, organized hospital infection control (IC) programs are widely implemented but are frequently under-resourced. Japan's fee-for-service payment system provides little incentive for hospitals to invest in IC. Without financial support for IC programs, hospital IC poses a heavy burden for Japanese health-care organizations. ${ }^{7}$

ICTs need to monitor the occurrence of infection and infectious disease, the causative bacteria and their antibiotic susceptibilities, and communicate this information with physicians. Another important role is to prevent the occurrence and spread of infection in the hospital

by ensuring instruction in skin disinfection, standard precautions and gown technique. ${ }^{8}$ Also, physician intervention to ensure the appropriate selection and use of antibiotics, especially broadspectrum antibiotics such as carbapenems, may be necessary to suppress the emergence of resistant strains.

In this study, we investigated how antibiotic use, the number of bacterial strains isolated and their antibiotic susceptibilities, and the use of disinfectant agents for hands in the hospital have changed since the ICT began to: (1) monitor if physicians are instructed in the use of broad-spectrum antibiotics; (2) check if measures for preventing the occurrence or spread of infectious disease are performed along with appropriate standard precautions; (3) provide rapid

${ }^{1}$ Division of Urology, Department of Organs Therapeutics, Kobe University Graduate School of Medicine, Kobe, Japan and ${ }^{2}$ Infection Control Team, Kobe University Hospital, Kobe, Japan

Correspondence: Dr K Shigemura, Division of Urology, Department of Organs Therapeutics, Kobe University Graduate School of Medicine, 7-5-1 Kusunoki-cho, Chuo-ku, Kobe 650-0017, Japan. 
communication with physicians in cases of bacteremia and (4) report the antibiograms in the hospital.

\section{MATERIALS AND METHODS}

\section{Bacteria}

Bacteria were isolated from April 2009 to March 2012 in the 920-bed Kobe University Hospital from all materials including urine, sputum, blood, nasal secretions or pus. The isolated bacteria were from $10^{5}$ or more colony forming units per $\mathrm{ml}$ in urine, one or more colony in $10 \mathrm{ml}$ of blood, one or more colony in sputum, one or more colony in nasal secretions and one or more colonies in pus.

\section{Susceptibility testing}

Antibiotic susceptibilities were tested and the results were interpreted and reported using the reference broth microdilution method, as described by the Clinical and Laboratory Standards Institute (CLSI) M7-A5 (2010; CLSI Document M100-S20). The MIC was defined as the lowest antimicrobial concentration that totally inhibited bacterial growth. Susceptibilities were evaluated by CLSI category. We tested bacterial strains against the following antimicrobial agents: ampicillin, piperacillin, amoxicillin-clavulanate combination, cefazolin, cefotiam, ceftriaxone, ceftazidime, cefmetazole, sulbactam/ cefoperazone, imipenem, gentamicin, levofloxacin, sulfamethoxazole/trimethoprim and minocycline. Antibiogram data were analyzed every 6 months. We divided our 3 years of observation into the following six periods: 2009-1st, from April 2009 to September 2009; 2009-2nd, from October 2009 to March 2010; 2010-1st, from April 2010 to September 2010; 2010-2nd, from October 2010 to March 2011; 2011-1st, from April 2011 to September 2011; and 20112nd, from October 2011 to March 2012. For quality control, Escherichia coli ATCC 25922, Staphylococcus aureus ATCC 29213, Pseudomonas aeruginosa ATCC 27853 and Enterococcus faecalis ATCC 29212 were used.

\section{ICT activity}

Our ICT was activated in its current configuration at the beginning of 2009 and consists of physicians, nurses and specialized laboratory technicians for bacteriology, and specialized pharmacologists for antibiotics. The physicians and nurses are qualified in IC. The main activities and roles of the ICT are as follows: (1) intervention for the use of broad-spectrum antibiotics as described below in physician interventions, (2) recommending the use of disinfectant agents for hands, (3) rapidly reporting to and communicating with physicians on bacteremia cases (within $24 \mathrm{~h}$ ), (4) reporting the antibiograms and the use of carbapenems in the hospital and (5) monitoring whether standard precaution measures were performed appropriately. We investigated how these works of the ICT affects antibiotic use, the number of bacterial strains isolated and their antibiotic susceptibilities over the past 3 years in the hospital, especially the comparison between the first 3 periods and the last 3 periods.

The amounts of hand disinfectant agents used in the hospital were calculated from the quantity shipped in the Department of Pharmacy from 2009 to 2012, totaled as the sum of chlorhexidine acetate with GOJO MHS (GOJO Japan K.K., Tokyo, Japan)

\section{Physician interventions}

Physician interventions were initiated in the current manner beginning in mid2010, involving pharmacologists specialized in antibiotics and physicians qualified in IC under the management of the ICT. The main purpose was to examine the use of antibiotics and check for inappropriate use or performance of blood culture tests, and contacting physicians about the performance of blood culture tests if necessary. Broad-spectrum antibiotics were targeted, specifically anti-Pseudomonas aeruginosa drugs such as piperacillin, tazobactam/piperacillin, ceftazidime, cefepime, cefozopran, ciprofloxacin, pazufloxacin, amikacin, tobramycin and gentamicin, and carbapenems such as meropenem, biapenem, doripenem and panipenem/betamiprone. The team provides instructions and physician interventions on appropriate use, for instance, to avoid insufficient dosing or unnecessary use. The frequency of use of these antibiotics and the number of physician interventions were also investigated.

\section{Statistical analyses}

Statistical analysis was conducted using linear regression analysis with PASW Statistics 17.0 software packages (for Windows; SPSS Inc., Chicago, IL, USA). The trends in bacteria isolation rate and annual consumption of antimicrobial agents were analyzed for each month and the susceptibility of isolated strains to antimicrobial agents was analyzed for each period. Statistical significance was established at the 0.05 level.

\section{RESULTS}

\section{Outcome of physician interventions}

The current system of physician interventions started in July 2010 with meetings held roughly once a week. There were 24 meetings in 2010 (from July to December) and 47 in 2011 (from January to December). In 2010, 1974 patient cases were discussed for appropriate use of antibiotics, and 4098 patients were discussed in 2011. The number of physicians interventions performed in 2010 was 193 cases compared with 491 cases in 2011. The details of the interventions are shown in Table 1. In brief, recommendations for blood culture tests were the most common intervention, followed by de-escalation of antibiotics $^{9-11}$ and change of antibiotics (Table 1).

\section{Blood culture tests}

To recommend an exact diagnosis for bacteremia and check the antibiotic susceptibilities of causative bacteria, the ICT recommended two sets of blood culture tests in cases suspicious for bacteremia and this was the most frequent intervention for physicians (48\% of total interventions in 2010 and 34\% in 2011) as shown in Table 2. There were a total of 63071 specimens including 19557 specimens in blood culture tests during the study period (Table 2). The ratio of performing two sets of blood culture tests in all blood culture tests showed an upward tendency from $58.1 \%$ in 2009 to $71.1 \%$ in 2010 and $80.3 \%$ in $2011(r=0.995, P=0.063, b=0.089)$. The isolation rate of organisms in blood culture tests was not significantly different, $12.6 \%$ in 2009 to $11.8 \%$ in 2010 and $10.0 \%$ in $2011(r=0.976$, $P=0.139, b=-0.733)$.

\section{Bacterial isolation and antibiograms}

A total of 13010 specimens of all organisms were isolated. The isolation rate of each organism showed no variation during the study period (Table 2). The antibiotic susceptibilities of methicillin sensitive S. aureus (MSSA) and E. faecalis, Enterobacteriae including E. coli, extended spectrum $\beta$-lactamase (ESBL)-producing E. coli, K. pneumoniae, Enterobacter cloacae and S. marcescens, and P. aeruginosa in every year is shown in Tables 3, 4 and 5, respectively. Susceptibility rates of E. faecalis to ampicillin significantly increased from $99.2 \%$ in 2009 to $100 \%$ in 2010 and $2011(r=0.864, P=0.026, b=1.875)$

\section{Table 1 Details of ICT interventions to physicians}

\begin{tabular}{lcc}
\hline & 2010 & 2011 \\
\hline Intervention (case number) & 193 & 491 \\
Objective number for intervention & 1947 & 4098 \\
Details of intervention (\%) & & \\
Blood culture (appropriate) performance & 48 & 34 \\
Antibiotic de-escaration & 10 & 14 \\
Change of antibiotics & 8 & 13 \\
Cancelation of antibiotics & 5 & 11 \\
Dose-up of antibiotics & 1 & 3 \\
Others & 28 & 25
\end{tabular}

Abbreviation: ICT, infection control team. 
Table 2 Trends of blood culture and organism isolation rates at the Kobe University Hospital, 2009-2011

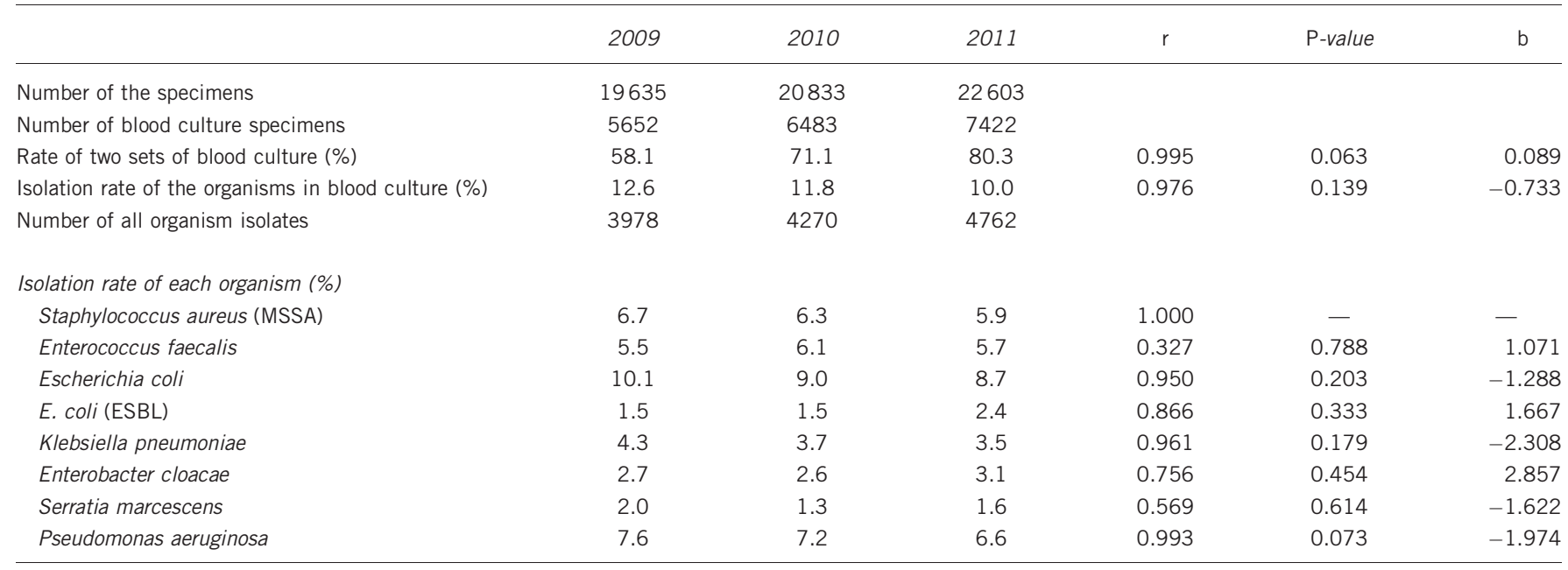

Abbreviations: $b$, regression coefficient; ESBL, extended spectrum $\beta$-lactamaset; $r$, correlation coefficient.

Table 3 Antibiotics susceptibility of Staphylococcus aureus (MSSA) and Enterococcus faecalis at the Kobe University Hospital, 2009-2011

\begin{tabular}{|c|c|c|c|c|c|c|c|c|c|}
\hline \multirow[b]{2}{*}{ Organism/antibiotic } & \multicolumn{6}{|c|}{ Antibiotics susceptibility rate (\%) } & \multirow[b]{2}{*}{ r } & \multirow[b]{2}{*}{ P-value } & \multirow[b]{2}{*}{$b$} \\
\hline & $2009-1 s t$ & 2009-2nd & $2010-1 s t$ & $2010-2 n d$ & 2011-1st & 2011-2nd & & & \\
\hline \multicolumn{10}{|l|}{ Staphylococcus aureus (MSSA) } \\
\hline Tested number of isolates & 141 & 152 & 172 & 268 & 157 & 201 & & & \\
\hline CEZ & 100 & 96.8 & 100 & 100 & 99.2 & 100 & 0.397 & 0.436 & 0.278 \\
\hline CTM & NT & 96.8 & 100 & 100 & NT & 100 & 0.795 & 0.205 & 0.406 \\
\hline IPM & 100 & 96.8 & 83.4 & 100 & 100 & 100 & 0.130 & 0.806 & 0.018 \\
\hline GM & 84.4 & 77.6 & 86.9 & 77.9 & 80.3 & 78.8 & 0.220 & 0.675 & -0.052 \\
\hline LVFX & 87.9 & 93.6 & 100 & 85.6 & 84.8 & 87.3 & 0.371 & 0.470 & -0.057 \\
\hline CLDM & 86.5 & NT & NT & 77.5 & 75.8 & 68.5 & 0.927 & 0.073 & -0.122 \\
\hline MINO & 96.5 & NT & NT & 97.7 & 97.7 & 94.5 & 0.226 & 0.774 & -0.146 \\
\hline ST & 100 & NT & NT & 100 & 99.2 & 100 & 0.478 & 0.522 & -1.167 \\
\hline TEIC & 100 & 100 & 100 & 100 & 100 & 100 & - & - & - \\
\hline VCM & 100 & 100 & 100 & 100 & 100 & 100 & - & - & - \\
\hline \multicolumn{10}{|l|}{ Enterococcus faecalis } \\
\hline Tested number of isolates & 119 & 158 & 168 & 243 & 174 & 176 & & & \\
\hline PCG & 92.2 & 97.7 & NT & NT & 97.5 & 100 & 0.699 & 0.301 & 2.447 \\
\hline ABPC & 99.2 & 99.2 & 100 & 100 & 100 & 100 & 0.864 & 0.026 & 18.750 \\
\hline IPM & 100 & 99.2 & 99.3 & 99.5 & NT & NT & 0.371 & 0.629 & -6.053 \\
\hline LVFX & 86.6 & 84.5 & 86.2 & 83.7 & 86.8 & 91.0 & 0.587 & 0.221 & 2.070 \\
\hline VCM & 100 & 100 & 100 & 100 & 100 & 100 & - & - & - \\
\hline
\end{tabular}

Abbreviations: ABPC, ampicillin; $b$, regression coefficient; CEZ, cefazolin; CLDM, clindamycin; CTM, cefotiam; GM, gentamicin; IPM, ipipenem; LVFX, levofloxacin; MINO, minocycline; NT, not tested; PCG, penicillin G; $r$, correlation coefficient; ST, sulfamethoxazole/trimethoprim; TEIC, teicoplanin; VCM, vancomycin.

The bold value is statistically significant.

(Table 3), while those of ESBL-producing E. coli to amoxicillinclavulanate combination significantly decreased from $67.6 \%$ in 2009-1st to $56.7 \%$ in 2011-2nd ( $r=0.961, P=0.009, b=-0.195$ ) (Table 4). Except for them, there were no other strains with lower susceptibilities in the most recent 1.5 years in this 3 -year investigation.

\section{Analysis of amount of disinfectant agents used for hands}

We checked the use of hand disinfectant agents to create an index for compliance for this standard precaution. The results are shown in Table 6. The total amount of disinfectant agents used for hands tended to increase year by year even though direct statistical analyses could not easily be performed.

\section{DISCUSSION}

The significance of IC and prevention has recently been broadly reconsidered after nosocomial infections, especially those involving resistant strains, became the focus of media reports on fatal cases or new resistant strains. ${ }^{12-14}$ Our hospital established an ICT in 2000 consisting of only one physician and one to two nurses; in 2009, this was expanded to include several specialized laboratory technicians 
Table 4 Antibiotics susceptibility of Enterobacteriaceae at the Kobe University Hospital, 2009-2011

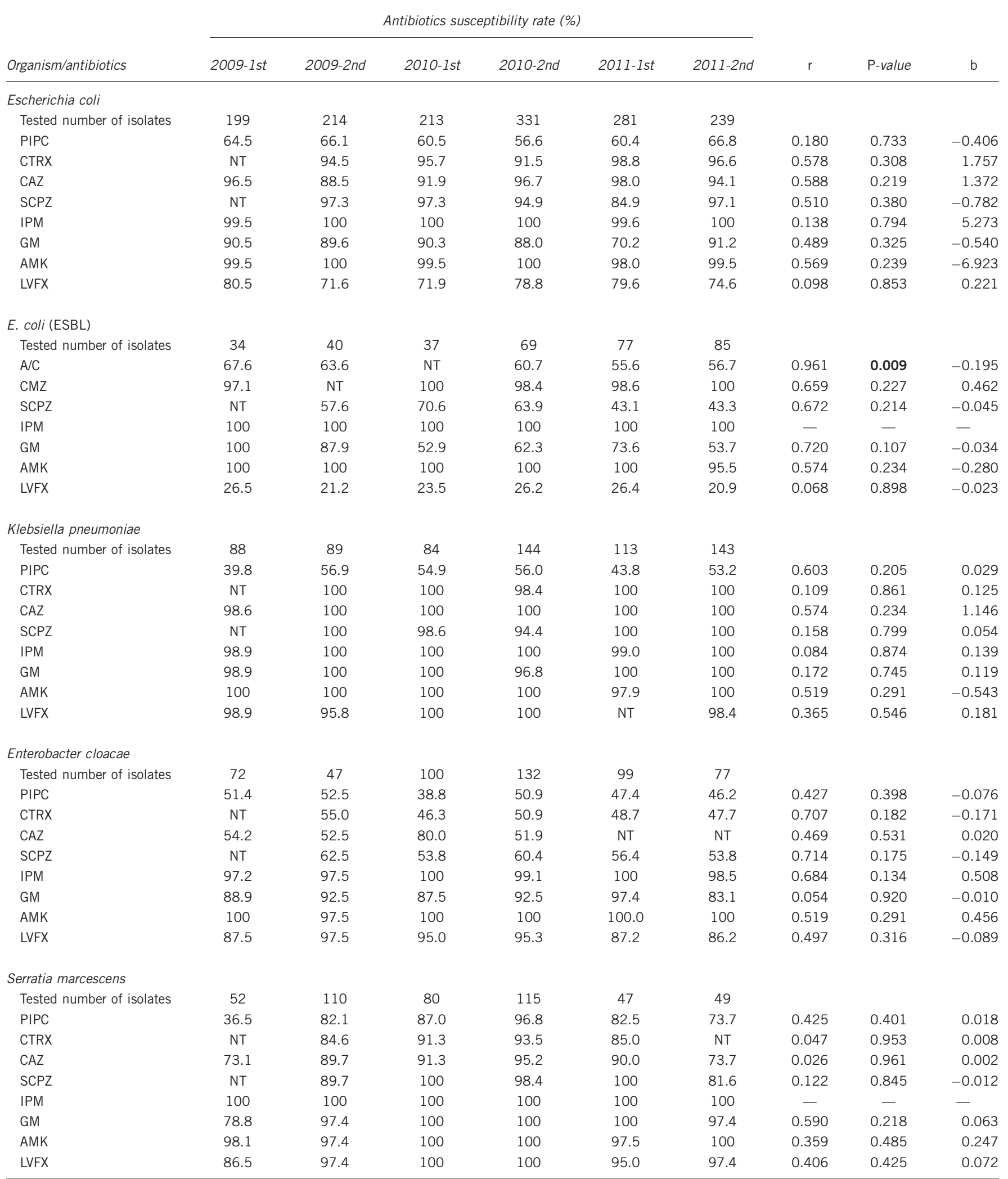

Abbreviations: AMK, amikacin; $b$, regression coefficient; CAZ, ceftazidime; CTRX, ceftriaxone; ESBL, extended spectrum $\beta$-lactamaset; GM, gentamicin; IPM, ipipenem; LVFX, levofloxacin; NT, not tested; PIPC, piperacillin; $r$, correlation coefficient; SCPZ, sulbactam/cefoperazone.

The bold value is statistically significant. 
Table 5 Antibiotics susceptibility of Pseudomonas aeruginosa at the Kobe University Hospital, 2009-2011

\begin{tabular}{|c|c|c|c|c|c|c|c|c|c|}
\hline \multirow[b]{2}{*}{ Organism/antibiotics } & \multicolumn{6}{|c|}{ Antibiotics susceptibility rate (\%) } & \multirow[b]{2}{*}{ r } & \multirow[b]{2}{*}{ P-value } & \multirow[b]{2}{*}{$b$} \\
\hline & $2009-1 s t$ & 2009-2nd & $2010-1 s t$ & $2010-2 n d$ & 2011-1st & 2011-2nd & & & \\
\hline \multicolumn{10}{|l|}{ Pseudomonas aeruginosa } \\
\hline Tested number of isolates & 184 & 185 & 286 & 376 & 255 & 273 & & & \\
\hline PIPC & 88.0 & 94.0 & 95.2 & 92.7 & 86.8 & 94.1 & 0.036 & 0.947 & -0.009 \\
\hline $\mathrm{P} / \mathrm{T}$ & NT & NT & 96.5 & 94.9 & 89.9 & 93.9 & 0.752 & 0.248 & -0.155 \\
\hline CFPM & 86.3 & 91.3 & 90.4 & 89.7 & 84.1 & 89.6 & 0.275 & 0.598 & -0.089 \\
\hline SCPZ & NT & 89.5 & 86.3 & 84.6 & 77.0 & 82.6 & 0.878 & 0.050 & -0.155 \\
\hline IPM & 79.9 & 83.3 & 84.7 & 82.8 & 70.9 & 77.8 & 0.620 & 0.189 & -0.110 \\
\hline MEPM & 85.7 & 88.0 & 81.3 & 86.8 & 85.5 & 78.7 & 0.591 & 0.217 & -0.148 \\
\hline GM & 79.9 & 93.0 & 88.7 & 91.8 & 88.0 & 88.0 & 0.190 & 0.718 & 0.037 \\
\hline AMK & 94.0 & 84.0 & NT & 86.1 & 86.8 & 85.4 & 0.411 & 0.492 & -0.105 \\
\hline LVFX & 89.1 & 96.0 & 96.2 & 94.4 & 96.0 & 100 & 0.713 & 0.112 & 0.180 \\
\hline CPFX & 90.3 & 80.7 & 79.9 & 83.1 & 83.3 & 84.6 & 0.203 & 0.700 & -0.049 \\
\hline AZT & 74.5 & NT & NT & 77.1 & 71.2 & 69.2 & 0.690 & 0.310 & -0.192 \\
\hline
\end{tabular}

Abbreviations: AMK, amikacin; AZT, aztoreonum; b, regression coefficient; CFPN, cefcapene; CPFZ, ciprofloxacin; GM, gentamicin; IPM, ipipenem; LVFX, levofloxacin; MEPM, meropenem; NT, not tested; PIPC, piperacillin; P/T, piperacillin/tazobactam; $r$, correlation coefficient; SCPZ, sulbactam/cefoperazone.

Table 6 Analysis of used amount of disinfectant agents for hands

\begin{tabular}{lcccc}
\hline & 2009 & 2010 & 2011 & 2012 \\
\hline GOJO MHS (L) & 309630 & 467910 & 525630 & 568320 \\
Chlorhexidine acetate (L) & 106000 & 232000 & 247000 & 391000 \\
Total (L) & 415630 & 699910 & 772630 & 959320 \\
\hline
\end{tabular}

for bacteriology, specialized pharmacologists for antibiotics, and physicians specialized in infection control and infectious diseases.

One of the roles of the ICT has been generally recognized as suppression of nosocomial infection, and to accomplish this goal the ICT reports the current status of bacteria isolated in the hospital, especially focusing on resistant strains such as ESBL-producing bacteria, as well as preventive measures used for suppressing the spread of infection such as instruction in hand disinfection. ${ }^{15-17}$ Our results show that the antibiogram of ESBL-producing $E$. coli resistant to amoxicillin-clavulanate combination decreased from 2009-1st to 2011-2nd. In addition, the isolated ratio of ESBL-producing E. coli in all E. coli tended to increase in the past three periods $(20.8 \%$ in 2010 2nd, $27.4 \%$ in 2011-1st and $35.6 \%$ in 2011-2nd) compared to the previous three periods $(17.1 \%$ in $2009-1$ st, $18.7 \%$ in $2009-2$ nd and $17.4 \%$ in 2010-1st), and the susceptibility of ESBL-producing E. coli to levofloxacin was $20.9-26.5 \%$ (Table 4). The ratio of ESBLproducing E. coli isolation was higher than that seen in European studies $(11 \%)^{18}$ and the susceptibilities to levofloxacin were similar to other studies from Japan. ${ }^{19}$ These facts provide significant data for preventing further increases in the isolation of resistant strains in hospitals and further decreases in antibiotic susceptibilities, two of the essential tasks of the ICT.

To limit the emergence of resistant strains or inhibit infection as soon as possible, physician interventions are also necessary in cases of inappropriate use of antibiotics, ${ }^{20,21}$ especially the unnecessary use of a broad-spectrum antibiotics and/or insufficient dosing over a long duration which may cause the emergence of resistant strains but not suppress infection. ${ }^{22,23}$ Our interventions involve reporting the total antibiotic use, the bacterial strains isolated and their antibiotic susceptibilities. The data showed that after ICT intervention, antibiotic use changed to appropriate dosages or to antibiotics with a narrow spectrum for the targeted bacteria (data not shown).
Our most frequent ICT intervention was the recommendation of two sets of blood culture tests to improve the diagnosis of bacteremia and help select appropriate antibiotic therapies based on the antimicrobial susceptibility results of the causative bacteria. ${ }^{24,25}$ As mentioned above for the guideline, a single set of blood culture tests may result in bacterial contamination and this might decrease the significance of the test and lead to inappropriate antibiotic use.

Our future task is to expand the role of the ICT hospital-wide. For this purpose, we will establish committees and hold conferences on topics of IC, infectious disease or antibiotics for all the workers in the hospital, not just physicians, including nurses, clinical technologists and pharmacologists, as well as physicians whose specialties are not as closely involved with infectious disease prevention or IC. Our mission is to promote the concept that IC requires the participation of all the workers in the hospital.

We would like to emphasize the limitations of our work. First, it is not easy to quantify the direct relationship between ICT activity and changes in the antibiograms from the statistical analyses. Second, the observation period is too short to draw definite conclusions. These limitations should be overcome by the quantification of ICT activity and statistical evaluation over a longer duration of observation.

In conclusion, since the introduction of an expanded ICT role, our results showed increased antibiotic susceptibilities in bacteria such as E. faecalis and the total amount of hand disinfectant agents tended to increase year by year even though direct statistical analyses could not be easily performed. Further observation may be necessary for a definitive evaluation of ICT activities.

\section{ACKNOWLEDGEMENTS}

We thank Gary Mawyer for English editing.

1 Carlet, J. et al. Society's failure to protect a precious resource: antibiotics. Lancet $\mathbf{3 7 8}$, 369-371 (2011).

2 Gordts, B. Models for the organisation of hospital infection control and prevention programmes. Clin. Microbiol. Infect. 11, S19-S23 (2005).

3 Neuhauser, M. M. et al. Antibiotic resistance among gram-negative bacilli in US intensive care units: implications for fluoroquinolone use. JAMA 289, 885-888 (2003). 
4 Venberghe, A. et al. Surveillance of hospital-acquired infections in an intensive care department-the benefit of the full-time presence of an infection control nurse. J. Hosp. Infect. 52, 56-59 (2002).

5 Niwa, T. et al. Outcome measurement of extensive implementation of antimicrobia stewardship in patients receiving intravenous antibiotics in a Japanese university hospital. Int. J. Clin. Pract. 66, 999-1008 (2012).

6 Yassi, A., Bryce, E. A., Maultsaid, D., Lauscher, H. N. \& Zhao, K. The impact of requiring completion of an online infection control course on health professionals' intentions to comply with infection control guidelines: a comparative study. Can. J. Infect. Dis. Med. Microbiol. 20, 15-19 (2009).

7 Sekimoto, M. et al. Impact of hospital accreditation on infection contro programs in teaching hospitals in Japan. Am. J. Infect. Control 36, 212-219 (2008)

8 Gould, I. M. Antibiotic policies and control of resistance. Curr. Opin. Infect. Dis. 15, 395-400 (2002)

9 Joung, M. K. et al. Impact of de-escalation therapy on clinical outcomes for intensive care unit-acquired pneumonia. Crit. Care 15, R79 (2011).

10 Shime, N., Satake, S. \& Fujita, N. De-escalation of antimicrobials in the treatment of bacteraemia due to antibiotic-sensitive pathogens in immunocompetent patients. Infection 39, 319-325 (2011).

11 Masterton, R. G. Antibiotic de-escalation. Crit. Care. Clin. 27, 149-162 (2011).

12 Chihara, S., Okuzumi, K., Yamamoto, Y., Oikawa, S. \& Hishinuma, A. First case of New Delhi metallo-beta-lactamase 1-producing Escherichia coli infection in Japan. Clin. Infect. Dis. 52, 153-154 (2011).

13 Yamamoto, T., Takano, T., Iwao, Y. \& Hishinuma, A. Emergence of NDM-1-positive capsulated Escherichia coli with high resistance to serum killing in Japan. J. Infect. Chemother. 17, 435-439 (2011).

14 Yamamoto, M. et al. Interspecies dissemination of a novel class 1 integron carrying blalMP-19 among Acinetobacter species in Japan. J. Antimicrob. Chemother. 66, 2480-2483 (2011)
15 Lee, S. O. et al. Reduced use of third-generation cephalosporins decreases the acquisition of extended-spectrum beta-lactamase-producing Klebsiella pneumoniae. Infect. Control Hosp. Epidemiol. 25, 832-837 (2004).

16 Goddard, S. \& Muller, M. P. The efficacy of infection control interventions in reducing the incidence of extended-spectrum $\beta$-lactamase-producing Enterobacteriaceae in the nonoutbreak setting: a systematic review. Am. J. Infect. Contro/ 39, 599-601 (2011).

17 D'Agata, E. M., Horn, M. A., Ruan, S., Webb, G. F. \& Wares, J. R. Efficacy of infection control interventions in reducing the spread of multidrug-resistant organisms in the hospital setting. PLoS. One 7, e30170 (2012).

18 Hawser, S. P. et al. Susceptibility of European Escherichia coli clinical isolates from intra-abdominal infections, extended-spectrum $\beta$-lactamase occurrence, resistance distribution, and molecular characterization of ertapenem-resistant isolates (SMART 2008-2009). Clin. Microbiol. Infect. 18, 253-259 (2012).

19 Yamamoto, A. Extended-spectrum $\beta$-lactamase (ESBL)-producing Escherichia coli is frequently detected as a pathogen of urinary tract infection in nursing home residents. Nihon Ronen Igakkai Zasshi 48, 530-538 (2011).

20 Perz, J. F. et al. Changes in antibiotic prescribing for children after a community-wide campaign. JAMA 287, 3103-3109 (2002).

21 Earnshaw, S. et al. European Antibiotic Awareness Day, 2008-the first Europe-wide public information campaign on prudent antibiotic use: methods and survey of activities in participating countries. Euro. Surveill. 14, 19280 (2009).

22 Levy, S. B. Antibiotic resistance: an ecological imbalance. Ciba. Found. Symp. 207 1-9 (1997).

23 Wenzel, R. P. \& Edmond, M. B. Managing antibiotic resistance. N. Engl. J. Med. 343, 1961-1963 (2000)

24 Towns, M. L., Jarvis, W. R. \& Hsueh, P. R. Guidelines on blood cultures. J. Microbiol. Imuunol. Infect. 43, 347-349 (2010).

25 Abe, T., Tokuda, Y., Ishimatsu, S. \& Birrer, R. B. Usefulness of initial blood cultures in patients admitted with pneumonia from anemergency department in Japan. J. Infect Chemother. 15, 180-186 (2009). 